HIGH-SPEED PHOTODIODES IN STANDARD CMOS TECHNOLOGY 


\author{
ANALOG CIRCUITS AND SIGNAL PROCESSING \\ Consuliting Editor: Mohammed Ismail. Ohio State University
}

Retated Thtes:

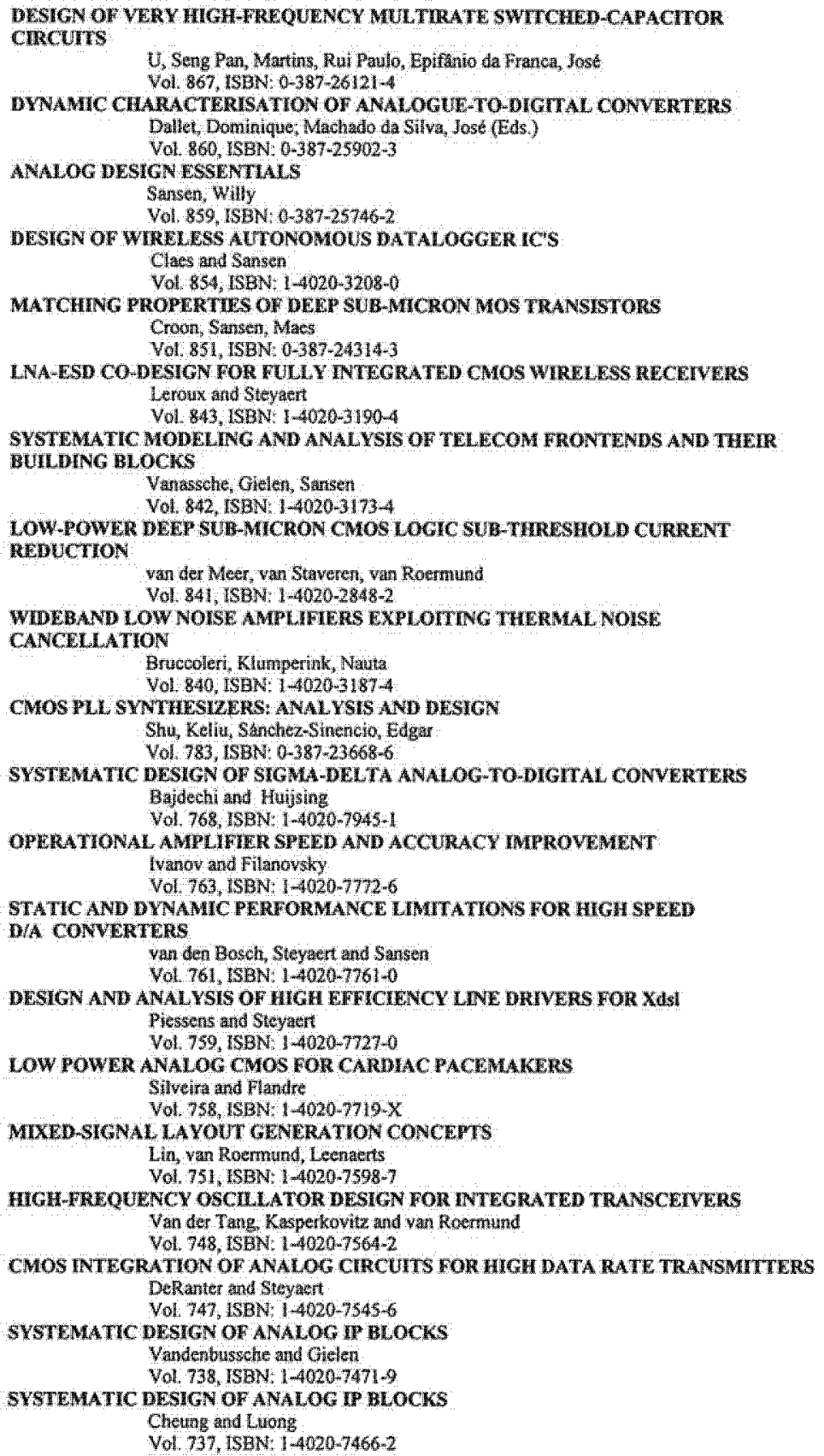




\section{HIGH-SPEED PHOTODIODES \\ IN STANDARD CMOS TECHNOLOGY}

by

Saša Radovanović

National Semiconductor,

The Netherlands

Anne-Johan Annema

University of Twente, Enschede,

The Netherlands

and

\section{Bram Nauta}

University of Twente, Enschede,

The Netherlands

Springer 
A C.I.P. Catalogue record for this book is available from the Library of Congress.

ISBN-10 0-387-28591-1 (HB)

ISBN-13 978-0387-28591-7 (HB)

ISBN-10 0-387-28592-X (e-book)

ISBN-13 978-0-387-28592-4 (e-book)

Published by Springer,

P.O. Box 17, 3300 AA Dordrecht, The Netherlands.

www.springer.com

Printed on acid-free paper

All Rights Reserved

(C) 2006 Springer

No part of this work may be reproduced, stored in a retrieval system, or transmitted in any form or by any means, electronic, mechanical, photocopying, microfilming, recording or otherwise, without written permission from the Publisher, with the exception of any material supplied specifically for the purpose of being entered and executed on a computer system, for exclusive use by the purchaser of the work.

Printed in the Netherlands. 


\section{Contents}

1 Introduction 1

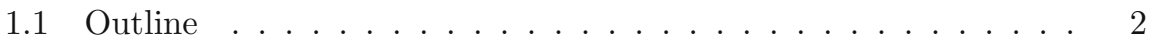

2 Short range optical interconnection $\quad 7$

2.1 Why optical interconnection? . . . . . . . . . . . . 7

2.1.1 Electrical and Optical Interconnection - Similarities . . . 8

2.1.2 Electrical and Optical Interconnection - Differences . . . . 9

2.2 Characteristics of light . . . . . . . . . . . . . . . . . . . 11

2.3 Optical fiber types . . . . . . . . . . . . . . . . . . . . 12

2.3.1 Single-mode fibers . . . . . . . . . . . . . 12

2.3.2 Multimode fibers . . . . . . . . . . . . . . 12

2.3.3 Plastic optical fibers . . . . . . . . . . . 16

2.4 High intensity light sources . . . . . . . . . . . . . . . . . . . . . . . . . . . . 16

2.4 Lasers . . . . . . . . . . . . . . . . . . 17

2.4.2 Light Emitting Diodes (LEDs) . . . . . . . . . . . 18

2.5 Photodetectors - introduction . . . . . . . . . . . . 18

2.5.1 Ideal photodetector . . . . . . . . . . . . . . 19

2.5.2 Absorption of light in silicon . . . . . . . . . . . 20

2.6 High-speed optical receivers in CMOS for $\lambda=850$ nm-literature overview . . . . . . . . . . . . . . . . . 24

2.6.1 Using standard CMOS technology . . . . . . . . . . . . 24 
2.6.2 CMOS technology modification . . . . . . . . . . 27

3 CMOS photodiodes for $\lambda=850 \mathbf{n m} \quad 33$

3.1 Introduction . . . . . . . . . . . . . . . . . . 34

3.2 Bandwidth of photodiodes in CMOS . . . . . . . . . . . . . 38

3.2.1 Intrinsic (physical) bandwidth . . . . . . . . . . . 38

3.2.2 Comparison between simulations and measurements . . . 61

3.2.3 N+/p-substrate diode . . . . . . . . . . . . 65

3.2.4 $\mathrm{P}+/$ nwell/p-substrate photodiode with low -resistance substrate in adjoined-well technology . . . . 66

3.3 Intrinsic (physical) photodiode bandwidth . . . . . . . . . . . . 70

3.4 Extrinsic (electrical) photodiode bandwidth . . . . . . . . . . 72

3.5 Noise in photodiodes . . . . . . . . . . . . . . . . 75

3.6 Summary and conclusions . . . . . . . . . . . . . . . . 75

4 High data-rates with CMOS photodiodes $\quad 79$

4.1 Introduction . . . . . . . . . . . . . . . . . . . . . 79

4.2 Transimpedance amplifier design . . . . . . . . . . . . . 81

4.2.1 Transimpedance amplifiers and extrinsic bandwidth . . . 82

4.2 .2 Impact of noise: BER . . . . . . . . . . . . . . 83

4.2.3 Noise of the TIA . . . . . . . . . . . . . . . . . 84

4.3 Photodiode selection . . . . . . . . . . . . . . 86

4.4 Equalizer design . . . . . . . . . . . . . . . . . . 88

4.5 Robustness on spread and temperature . . . . . . . . . . . . 91

4.6 Experimental results . . . . . . . . . . . . . . . . . . . 95

4.6.1 Circuit details and measurement setup . . . . . . . . . . 95

4.6.2 Optical receiver performance without equalizer . . . . . . 97

4.6.3 Optical receiver performance with equalizer . . . . . . . 97

4.6.4 Robustness of the pre-amplifier: component spread . . . . 99

4.6.5 Robustness of the pre-amplifier: diode spread . . . . . . . 100

4.7 Conclusions . . . . . . . . . . . . . . . . . . . 102

5 Bulk CMOS photodiodes for $\lambda=400 \mathrm{~nm} \quad \mathbf{1 0 5}$

5.1 Introduction . . . . . . . . . . . . . . . . 106

5.2 Finger nwell/p-substrate diode in adjoined-well technology . . . . 107

5.3 Finger $\mathrm{n}+/$ nwell $/ \mathrm{p}$-substrate diode . . . . . . . . . . . . . . . 109

5.3.1 Time domain measurements . . . . . . . . . . . 113 
5.4 Finger $\mathrm{n}+/ \mathrm{p}$-substrate photodiode in separate-well technology . . . . . . . . . . . . . 115

5.5 Finger $\mathrm{p}+/$ nwell $/ \mathrm{p}$-substrate in adjoined-well technology . . . . . . . . . . . . . . . . 116

5.5.1 Time domain measurements . . . . . . . . . . . . . . 117

$5.6 \mathrm{p}+$ /nwell photodiode . . . . . . . . . . . . . . . . 118

5.7 Conclusion . . . . . . . . . . . . . . . . . . . 119

6 Polysilicon photodiode $\quad 123$

6.1 High-speed lateral polydiode . . . . . . . . . . . . . . . . . 123

6.1.1 Pulse response of the poly photodiode . . . . . . . . . 127

6.1.2 Diffusion current outside the depletion region . . . . . . . 130

6.1.3 Frequency characterization of the polysilicon photodiode . . . . . . . . . . . 131

6.2 Noise in polysilicon photodiodes . . . . . . . . . . . . . . . . . . 134

6.2.1 Dark leakage current in the polysilicon diode . . . . . . . 134

6.3 Time domain measurements . . . . . . . . . . . . . . . . 135

6.4 Quantum efficiency and sensitivity . . . . . . . . . . . . 138

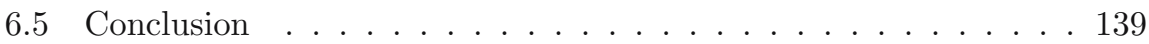

7 CMOS photodiodes: generalized $\quad 143$

7.1 Introduction . . . . . . . . . . . . . . . . . . . . . 143

7.2 Generalization of CMOS photodiodes . . . . . . . . . . . . 145

7.3 Device layer - photocurrent amplitude . . . . . . . . . . . . 146

7.3.1 Device layer - photocurrent bandwidth . . . . . . . . . . 146

7.3.2 Substrate current-photocurrent amplitude . . . . . . . . . 148

7.3.3 Substrate current-photocurrent bandwidth . . . . . . . . . 150

7.3.4 Depletion region current . . . . . . . . . . . . 152

7.3.5 Depletion region - photocurrent bandwidth . . . . . . 153

7.3.6 Total photocurrent . . . . . . . . . . . . 153

7.4 Analog equalization . . . . . . . . . . . . . . . . 155

7.5 Summary and Conclusions . . . . . . . . . . . . . . . . 156

8 Conclusions $\quad 159$

8.1 Conclusions . . . . . . . . . . . . . . . . . . . 159 